\title{
Comparison between Erigo tilt-table exercise and conventional physiotherapy exercises in acute stroke patients: a randomized trial
}

\author{
Suraj Kumar ${ }^{1 *}$ (D, Ramakant Yadav² and Aafreen ${ }^{1}$
}

\begin{abstract}
Background: Stroke is a common, serious, and disabling health-care problem throughout the world. Although great advances have been made in acute stroke management, the most of post-stroke care to reduce a patient's dependency relies on rehabilitation.

Purpose: To compare the effectiveness of exercises using an Erigo tilt-table and conventional physiotherapy in the rehabilitation of acute stroke patients.

Methods: A total of 110 acute stroke patients (age $51.08 \pm 7.48$ years, $8.69 \pm 4.62$ days after stroke) were assigned randomly into two groups, 55 in each for 30 days of conventional physiotherapy (Group A) or Erigo tilt-table (Group B) rehabilitation. The National Institutes of Health Stroke Scale (NIHSS), Mini-Mental Scale Examination (MMSE), Modified Ashworth Scale were used to measure muscle tone, quality of life (QOL) and muscle strength (MMT), Affected upper (UE) and lower limb (LE) outcomes were assessed at baseline (day 0), after day 30 of the intervention and on 90th day of follow up. Repeated measures ANOVA followed by a Bonferroni post-hoc test and independent Student's t-test were used for statistical analysis to evaluate the improvement in outcome variables within and between the groups.

Results: Both the treatments were effective. Notably, Group B patients showed a significant improvement in both $\mathrm{QOL}(p<0.001)$ and lower limb strength $(p=0.030)$ at day 90 and muscle tone $(p=0.011)$ at day 30 compared to Group A.

Conclusion: Both the groups improved with time but the Erigo tilt-table group experienced greater improvement in QOL, NIHSS and muscle strength of the lower limb. Thus, Erigo tilt-table can be used for early rehabilitation of acute hemiplegic patients and improving their quality of life and motor system, resulting in better functional performances.
\end{abstract}

Keywords: Physiotherapy, Quality of life, Stroke, Tilt-table

\footnotetext{
* Correspondence: surajdr2001@yahoo.com

'Department of Physiotherapy, UPUMS, Saifai, Etawah, UP 206130, India

Full list of author information is available at the end of the article
}

(c) The Author(s). 2020 Open Access This article is distributed under the terms of the Creative Commons Attribution 4.0 International License (http://creativecommons.org/licenses/by/4.0/), which permits unrestricted use, distribution, and reproduction in any medium, provided you give appropriate credit to the original author(s) and the source, provide a link to the Creative Commons license, and indicate if changes were made. The Creative Commons Public Domain Dedication waiver (http://creativecommons.org/publicdomain/zero/1.0/) applies to the data made available in this article, unless otherwise stated. 


\section{Introduction}

Stroke is a major cause of death and disability [1], and is often associated with negative secondary complications that might postpone or prevent recovery [2]. Early mobilization averts such negative effects and promotes recovery $[3,4]$. However, it is challenging as many patients are severely impaired and are non-cooperative. Mobilization using an Erigo tilt-table is a well-tolerated method of mobilization and can be considered a safe system of early mobilization of patients with severe brain injuries and stroke $[5,6]$. Patients in vegetative or minimally conscious state tolerate greater degrees of head-up tilt better with simultaneous leg movement [7]. Blood pressures and heart rate can be stabilized better with treatment with passive leg movements [8]. Moreover, an intensive cyclic leg loading verticalization protocol, started in the acute stages of a severe acquired brain injury, improves the short-term and long-term functional and neurological outcome of patients with disorder of consciousness [9].

The Erigo FES (Functional Electrical Stimulation) is fully synchronized with robotic leg movements. Thus, the cyclic FES to the leg muscles can effectively induce venous return, thereby helping to improve cerebral blood flow and to maintain the blood pressure of individuals with spinal cord injury under orthostatic stress $[10,11]$.

Neuro-rehabilitation plays a central role in successfully reducing the long-term effects of stroke and achieving optimal functional recovery for community re-integration. Neuroplasticity is the basic mechanism underlying improvement in functional outcome after stroke [12]. Therefore, an important goal of rehabilitation of stroke patients is the effective use of neuroplasticity for functional recovery. Thus, high-dose intensive training and repetitive practice of specific functional tasks are important for recovery after stroke.

The electromechanical/robotic devices that ensure automation of lower limb movements during locomotion were developed to help the physiotherapists by increasing the safety, intensity, and standardization of non-robotic body weight supported treadmill training, generate complex multisensory stimulation, offer extensive extrinsic biofeedback to the patient, and reduce the working costs [13].

The robotic Erigo tilt-table consists of a stretcher that can be tilted between $0^{\circ}-80^{\circ}$ and footplates with integrated springs for leg loading. Footplates do steppinglike movements. Training on the Erigo combines mobilization out of bed, body verticalization, and rhythmic leg movement with cyclic loading thus contributing to the prophylaxis of secondary complications caused by a prolonged period of immobility. The benefits offered by the Erigo compared to mobilization using a conventional tilt table are as follows:

- Passive movement of legs combined with verticalization of the patient
- A load is alternately applied and removed from the patient's legs

- The leg movement can be adapted separately for the patient's right and left leg according to his needs ([14], Erigo User Manual Part-1).

The most important advantage of using robot technology in rehabilitation is to deliver high-dosage and highintensity training.

Prolonged inactivity leads to deconditioning and complications appear during the first days of bed rest and worsen the devastating neurological injury. Therefore, early intervention by rehabilitation protocols should begin, to reduce the prolonged bed rest complications, stimulate the afferent sensory system and to reduce spasticity. Although earlier studies on rehabilitation with robotics proved to be beneficial, there are very few clinical studies available on acute stroke patients and its comparison with conventional physiotherapy. Thus, this study hypothesized that Erigo tilt-table therapy will be more effective than conventional physiotherapy for early mobilization and rehabilitation in acute stroke patients.

\section{Methods and materials Subjects}

The participants included in this study are post-stroke hemiparesis subjects referred to the Department of Neurology, ' $X$ ' aged 30-60 years either male or female diagnosed clinically by a neurologist with ischemic or hemorrhagic stroke, within 7-28 days of onset and with scores on the National Institutes of Health Stroke Scale (NIHSS) between 11 and 22. The present study has the approval of the Institutional Local Ethical Committee and informed consent was obtained from all the participants so that patients understood the purpose of the study. Subjects were excluded from the study if they had a metal implant, recurrent stroke, chronic renal failure, cognitive and speech problem, hemiplegia due to nonvascular causes (malignancy, infections, tumours, brain injury, etc.), sensation loss in the lower extremity, or poor sitting balance.

\section{Procedure}

The subjects were randomized into two groups by lottery method [15], Group A for conventional physiotherapy and Group B for robotic Erigo tilt-table rehabilitation. One hundred forty folded papers of the same shape and size were marked either Group A (70) or Group B (70) and were kept in a box. Paper drawn by the patient allocated the mode of treatment. The allocation was concealed and the assessor was blinded to group allocation. After group allocations, respective subjects' were treated either with conventional physiotherapy or robotic tilt- table therapy. Both treatments were 
given as individual treatment by the same physiotherapist for 30 regular days (except Sundays) and reassessment was done after 30 days and on follow up at 90th day. The duration of each treatment session was about 50 to $60 \mathrm{~min}$ per day. The demographic characteristics such as age, weight, height, BMI (body mass index), the side affected, and the onset of stroke (duration) of the two treatment groups were assessed at baseline before randomization. Similarly, outcome variables such as muscle strength (upper limb and lower limb), NIHSS, Ashworth, MMSE and QOL were also assessed by same tester and same physiotherapist supervising the test procedure at baseline (day 0 ) as well as at the end of treatment (day 30) and on follow- up (day 90). During 3090 days, subjects were asked to perform a home-exercise program. All the patients were provided with the homeexercise booklet which includes written instructions and figures. Subjects were once asked to perform these exercises under the supervision of physiotherapists and then to continue at home on their own or with the help of attendants. During this period the patients were contacted every 15 days either at the hospital or telephonically to determine whether they are performing the exercises properly or not.

Test and re-test of two groups were conducted in the same place in the same environment. Before experimentation, all subjects were well taught about the measurement variables and their outcomes. The subjects were also informed about experimental risks if any. All subjects were allowed to take treatment for their comorbid conditions such as hypertension, dyslipidemia, hypothyroidism, the cardiac problem in both the conditions under the supervision of neurologists. No other treatment was allowed other than those mentioned above.

\section{Training protocols}

\section{Conventional physiotherapy (Group A) [16]}

All the exercises were done for 10 repetitions, 2 sets with $10 \mathrm{~s}$ hold once a day under the supervision of the physiotherapist which includes the following:

- Full range of motion (ROM) exercises - passive and active-assisted range of motion exercises for the upper limb including shoulder (flexion, extension, abduction and adduction), elbow (flexion and extension), forearm (supination and pronation), wrist (flexion, extension, radial and ulnar deviation), and for the lower limb including hip (flexion, extension, abduction and adduction), knee (flexion and extension), ankle (dorsiflexion, plantar flexion, eversion and inversion).

- For spasticity management - Positioning of the limb, prolonged icing, brushing, gentle stroking, and gentle tapping [17-19]
- Common mat activities including turning from supine to side-lying to prone and vice versa, prone to prone on an elbow, prone on elbow to prone on hand; prone on hand to quadruped; quadruped to kneeling; kneeling to half-kneeling; half kneeling to standing with support; standing with support to the standing without support.

- Bridging exercises.

- Prolonged and gradually progressive stretching of hamstrings, calf, and wrist.

- Strengthening exercises included isometrics of the back, quadriceps, and gripping exercises.

- Gentle and controlled weight-bearing exercises.

- Balance and coordination exercises.

\section{Erigo tilt-table therapy (Group B)}

Erigo tilt-table therapy was administered according to the following protocol. The patient received a treatment session of $40 \mathrm{~min}, 6$ times per week [20] for about 4 weeks [21] (Table 1).

The Robotic tilt table exercise session was followed by a 15 min exercise program for upper extremities which including range of motion, strengthening and stretching exercises of shoulder, elbow, wrist, and fingers.

No specific gait training was administered to both the groups, but ambulation was offered to all patients when they were able to ambulate.

\section{Outcome variables}

The assessment of QOL was done according to the SF36 assessment tool [22]. It is a multipurpose, selfadministered, short-form (SF) health survey with 36 questions that measure generic health status in the general population. These questions consist of physical functioning, role functioning (physical and emotional), body pain, general health, vitality, social functioning, and, mental health. Response choices are numbered from left to right, starting with 1 . The maximum scores obtained from 36 questions where 149 which represents best QOL whereas minimum score 36 represents the worst [23]. The scale was used earlier in acute stroke patients [24, 25].

Muscle strength was measured by the MRC (Medical Research Council) classification of Manual Muscle Testing (MMT). Overall upper limb and lower limb muscle strength were recorded. For the upper limb, MMT was performed for shoulder flexion, shoulder extension, shoulder abduction, shoulder adduction, shoulder external rotation, shoulder internal rotation, elbow flexion, elbow extension, forearm pronation, forearm supination, wrist palmar flexion, wrist dorsal flexion, ulnar deviation, and radial deviation. The average score of these measures was considered as overall upper limb muscle strength. Similarly for the lower limb, MMT was done 
Table 1 The exercise protocol on Robotic Erigo tilt-table

\begin{tabular}{ll}
\hline Phase I (1st week) & At $30^{\circ}$ angle for 40 min with 1-min hold after every 12 min at $0^{\circ}$ angle \\
Phase II (2nd and 3rd weeks) & At $50^{\circ}$ angle for 40 min with 1-min hold after every 12 min at $0^{\circ}$ angle \\
Phase III (4th week) & At $75^{\circ}$ angle for 40 min with 1-min hold after every 12 min at $0^{\circ}$ angle \\
\hline
\end{tabular}

for hip flexion, hip extension, hip abduction, hip adduction, hip external rotation, hip internal rotation, knee flexion, knee flexion with leg external rotation, knee flexion with leg internal rotation, knee extension, ankle plantar flexion, ankle plantar flexion (soleus), foot dorsiflexion and inversion, foot inversion, foot eversion with plantar flexion and foot eversion with dorsiflexion. The average score of these measures was recorded as overall lower limb muscle strength. The positioning of the patients for performing MMT was according to standard norms and was also used earlier in a published study $[26,27]$.

The National Institutes of Health Stroke Scale or NIH Stroke Scale (NIHSS) is a tool used by healthcare providers to objectively quantify the impairment caused by a stroke. The NIHSS is composed of 11 items, each of which scores a specific ability between a 0 and 4 . For each item, a score of 0 typically indicates a normal function in that specific ability, while a higher score is indicative of some level of impairment. The individual scores from each item are summed to calculate a patient's total NIHSS score. The greatest possible score is 42 , with the lowest score being 0 [28].

The Mini-Mental State Examination (MMSE) is a 30point questionnaire used extensively in clinical and research settings to measure cognitive impairment. Administration of the test takes between 5 and $10 \mathrm{~min}$ and examines function including registration (repeating named prompts), attention and calculation, recall, language, ability to follow simple commands and orientation. Any score greater than or equal to 24 points (out of 30) indicates a normal cognition. Below this, scores can indicate severe ( $\leq 9$ points), moderate (10-18 points) or mild (19-23 points) cognitive impairment [29].

The Modified Ashworth Scale is a 6-point rating scale measuring muscle tone with ratings from 0 indicating no increase in tone to 5 indicating limb rigid in flexion or extension [30]. For the upper limb, the scores of shoulder adductors, elbow flexors, and wrist flexors were recorded and for the lower limb scores of hip adductors, knee extensors and ankle plantar flexors were recorded. The average of these readings considered as the global score [31].

\section{Statistical analysis}

Continuous data were summarized by mean and standard deviation (SD) whereas discrete (categorical) data were described by number (n) and percentage (\%). Two independent groups compared by independent Student's t-test. Groups were also compared by repeated measures two factors (Time*Group) analysis of variance (ANOVA) and the significance of mean difference within (intra) and between (inter) the groups were calculated by Bonferroni post hoc test for multiple contrasts. Categorical variables were compared by the chi-square $\left(\mathrm{x}^{2}\right)$ test. A two-tailed $(\alpha=2) \quad p<0.05$ was considered statistically significant.

\section{Results}

A total of 133 patients were assigned out of 365 patients enrolled from the Department of Neurology from May 2017 to September 2018. Twenty-three patients dropped out for different reasons (see Fig. 1). Of these 110 patients who completed the study, 55 were treated with conventional therapy (Group A) and 55 with Erigo tilttable (Group B) presented in Fig. 1. The mean age of the 110 patients was $51.08 \pm 7.48$ years. In 54 patients, stroke affected the right hemisphere. Patient demographic parameters are presented in Table 2. The training was started on average $8.69 \pm 4.62$ days after the stroke.

\section{Demographic characteristics}

The baseline demographic characteristics of the two groups are summarised in Table 2. On comparing, the baseline demographic characteristics were found similar $(p>0.05)$ between the two groups (Table 2) suggesting that both the groups were demographically matched and the outcomes would not be influenced by these parameters.

\section{Outcome measures}

The pre and post outcome measures of two groups is summarised in Table 3 and also depicted in Fig. 2. Comparison of the outcome measures of two groups over the periods (time), using ANOVA showed significant effect of groups on QOL $(F=11.77, p=0.001)$, MMT (UE) $(F=4.36, p=$ $0.039)$, MMT (LE) $(F=6.15, p=0.015)$ and NIHSS $(F=$ 6.05, $p=0.015)$, and also periods on QOL $(F=310.36, p<$ $0.001)$, MMT (UE) $(F=701.26, p<0.001)$, MMT (LE) $(F=$ 546.51, $p<0.001)$, NIHSS $(F=1517.30, p<0.001)$, MMSE $(F=617.73, p<0.001)$ and Ashworth $(F=37.31, p<0.001)$. Furthermore the interaction effect of both groups and periods (Groups"Time) on QOL ( $F=17.06, p<0.001)$, MMT (LE) $(F=3.62, p=0.028)$ and Ashworth $(F=3.11, p=$ 0.047) were also found significant (Fig. 2).

Further, for each outcome measure, comparing the difference in mean across the periods (intragroup), Bonferroni 


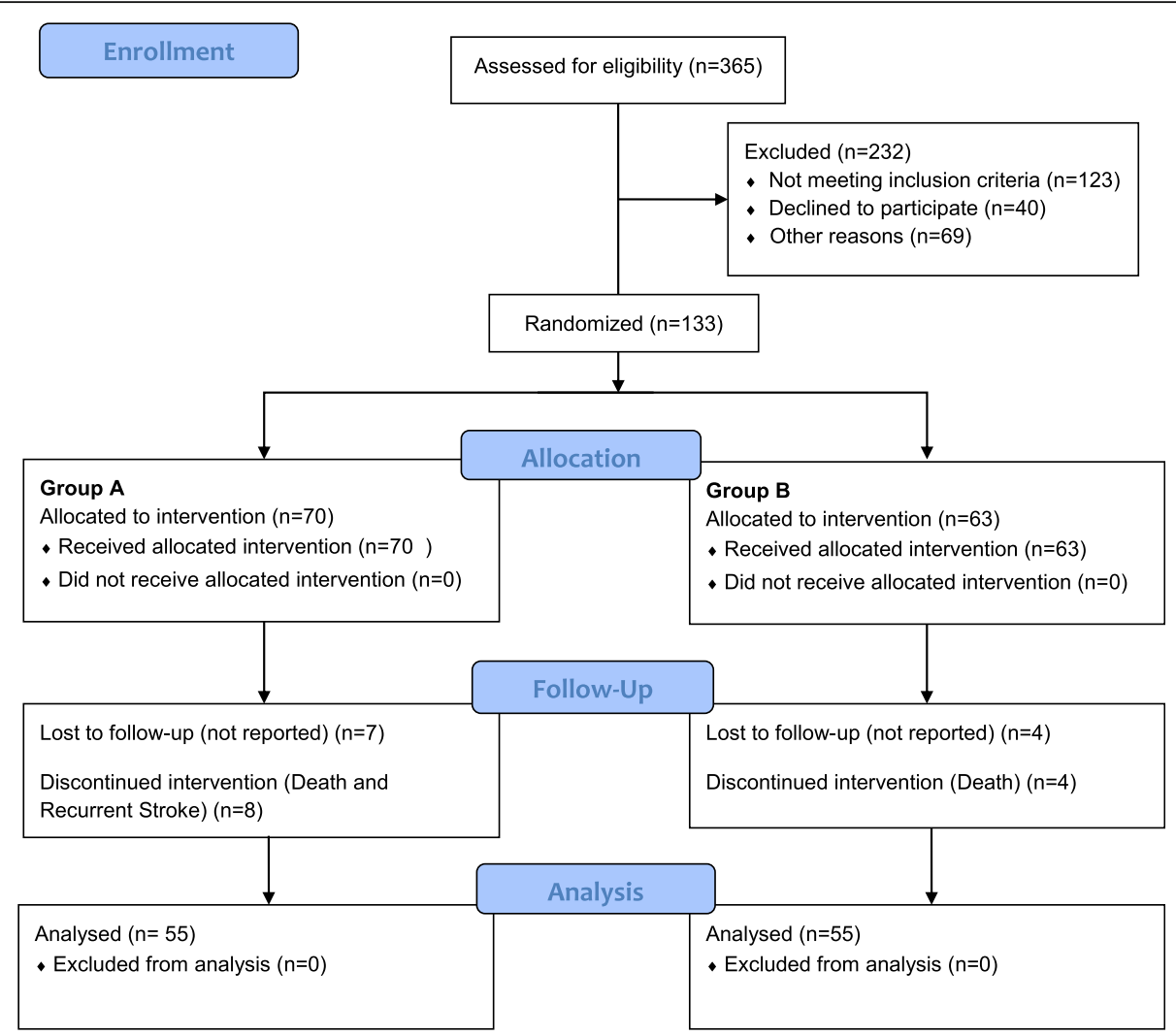

Fig. 1 The flow diagram shows the number of patients in the treatment groups

Table 2 Demographic characteristics (Mean \pm SD) of two

\begin{tabular}{|c|c|c|c|}
\hline Demographic characteristics & $\begin{array}{l}\text { Group A } \\
(n=55)(\%) \\
(\text { mean }+/-S D)\end{array}$ & $\begin{array}{l}\text { Group B } \\
(n=55)(\%) \\
\text { (mean }+/-S D)\end{array}$ & $P$ value \\
\hline Age (yrs) & $51.36 \pm 8.10$ & $50.80 \pm 6.86$ & 0.695 \\
\hline \multicolumn{4}{|l|}{ Sex: } \\
\hline Male & $30(54.5)$ & $30(54.5)$ & \multirow[t]{2}{*}{1.000} \\
\hline Female & $25(45.5)$ & $25(45.5)$ & \\
\hline Weight $(\mathrm{kg})^{\mathrm{a}}$ & $65.62 \pm 10.13$ & $63.93 \pm 7.14$ & 0.314 \\
\hline Height $(m)^{a}$ & $1.61 \pm 0.09$ & $1.60 \pm 0.09$ & 0.809 \\
\hline $\mathrm{BMI}(\mathrm{kg} / \mathrm{m} 2)^{\mathrm{a}}$ & $25.40 \pm 3.72$ & $24.89 \pm 2.46$ & 0.395 \\
\hline \multicolumn{4}{|l|}{ Side affected: } \\
\hline Right & $31(56.4)$ & $25(45.5)$ & \multirow[t]{2}{*}{0.252} \\
\hline Left & $24(43.6)$ & $30(54.5)$ & \\
\hline \multicolumn{4}{|l|}{ Lesion: } \\
\hline Haemorrhagic & $33(60.0)$ & $27(49.1)$ & \multirow[t]{2}{*}{0.251} \\
\hline Ischemic & $22(40.0)$ & $28(50.9)$ & \\
\hline $\mathrm{SBP}(\mathrm{mmHg})^{\mathrm{a}}$ & $136.18 \pm 16.50$ & $132.18 \pm 15.95$ & 0.199 \\
\hline $\mathrm{DBP}(\mathrm{mmHg})^{\mathrm{a}}$ & $86.91 \pm 9.60$ & $83.82 \pm 8.92$ & 0.083 \\
\hline
\end{tabular}

Group A Conventional physiotherapy, Group $B$ Erigo tilt-table, $B M I$ Body mass index, $S B P$ Systolic blood pressure, $D B P$ Diastolic blood pressure ${ }^{\mathrm{a}}$ mean test showed significant $(p<0.001)$ increase in QOL, MMT (UE), MMT (LE), MMSE and Ashworth while significant $(p<0.001)$ decreases in NIHSS at both day 30 and day 90 were noted compared to day 0 in both groups (except Ashworth in Group B) (Table 3). Furthermore, in both groups, QOL, MMT (UE), MMT (LE) and MMSE also showed significant $(p<0.001)$ increase while NIHSS showed significant $(p<0.001)$ decrease at day 90 as compared to day 30 .

Similarly, for each outcome measure, comparing the mean between the groups (intergroup), the Bonferroni test showed similar $(p>0.05)$ mean between the groups at day 0 indicating all outcome measures comparable (Table 3). However, at day 90, both QOL $(p<0.001)$ and MMT (LE) $(p=0.030)$ were found significantly different and higher in Group B as compared to Group A. In contrast, at day 90 , NIHSS was significantly lower $(p=$ 0.035) in Group B compared to Group A.

\section{Net improvement}

To determine the efficacy of one group over the other, the pre -to post- change in outcome measures were done and compared by independent Student's t-test and summarised in Table 4. Analysis using Student's t-test showed significant improvement in both QOL $(p<$ $0.001)$ and MMT (LE) $(p=0.030)$ of Group B as compared to Group A at day 90. Furthermore on day 30, 
Table 3 Outcome measure score (Mean \pm SD) of two groups over the periods

\begin{tabular}{|c|c|c|c|}
\hline Outcome measure & Day 0 & Day 30 & Day 90 \\
\hline \multicolumn{4}{|l|}{ QOL: } \\
\hline Group A & $75.45 \pm 6.59$ & $83.20 \pm 9.41^{a}$ & $89.84 \pm 11.74^{\mathrm{ab}}$ \\
\hline Group B & $77.71 \pm 8.69$ & $87.58 \pm 9.93^{\mathrm{a}}$ & $100.47 \pm 11.97^{\mathrm{ab}}$ \\
\hline$P$ value $\#$ & 1.000 & 0.321 & $<0.001$ \\
\hline \multicolumn{4}{|l|}{ MMT (UE): } \\
\hline Group A & $0.82 \pm 0.90$ & $2.16 \pm 0.96^{\mathrm{a}}$ & $2.93 \pm 0.88^{\mathrm{ab}}$ \\
\hline Group B & $1.17 \pm 0.86$ & $2.42 \pm 0.93^{\mathrm{a}}$ & $3.31 \pm 0.90^{\mathrm{ab}}$ \\
\hline$P$ value & 0.658 & 1.000 & 0.463 \\
\hline \multicolumn{4}{|l|}{ MMT (LE): } \\
\hline Group A & $1.25 \pm 1.04$ & $2.47 \pm 1.01^{\mathrm{a}}$ & $3.36 \pm 0.89^{\mathrm{ab}}$ \\
\hline Group B & $1.42 \pm 0.98$ & $2.88 \pm 0.83^{\mathrm{a}}$ & $3.90 \pm 0.54^{\mathrm{ab}}$ \\
\hline$P$ value & 1.000 & 0.241 & 0.030 \\
\hline \multicolumn{4}{|l|}{ NIHSS: } \\
\hline Group A & $12.53 \pm 1.59$ & $6.78 \pm 2.11^{\mathrm{a}}$ & $4.07 \pm 2.07^{\mathrm{ab}}$ \\
\hline Group B & $11.95 \pm 1.45$ & $6.20 \pm 2.00^{a}$ & $2.96 \pm 1.99^{\mathrm{ab}}$ \\
\hline$P$ value ${ }^{\#}$ & 1.000 & 1.000 & 0.035 \\
\hline \multicolumn{4}{|l|}{ MMSE: } \\
\hline Group A & $15.22 \pm 4.45$ & $22.00 \pm 3.59^{a}$ & $24.33 \pm 2.93^{\mathrm{ab}}$ \\
\hline Group B & $15.80 \pm 4.10$ & $22.15 \pm 3.46^{\mathrm{a}}$ & $24.42 \pm 2.81^{\mathrm{ab}}$ \\
\hline$P$ value $^{\#}$ & 1.000 & 1.000 & 1.000 \\
\hline \multicolumn{4}{|l|}{ Ashworth: } \\
\hline Group A & $0.09 \pm 0.29$ & $0.56 \pm 0.54^{\mathrm{a}}$ & $0.64 \pm 0.62^{\mathrm{ab}}$ \\
\hline Group B & $0.11 \pm 0.31$ & $0.33 \pm 0.51^{\mathrm{a}}$ & $0.45 \pm 0.57^{b}$ \\
\hline$P$ value & 1.000 & 0.181 & 0.793 \\
\hline
\end{tabular}

Group A Conventional physiotherapy, Group B Erigo tilt-table, QOL Quality of life, MMT (UE) Manual muscle testing (upper extremity) or overall upper limb strength, MMT (LE) Manual muscle testing (lower extremity) or overall lower limb strength, NIHSS National institute of health stroke scale, MMSE Minimental state examination, Ashworth Ashworth scale-6

${ }^{a} p<0.001$ - as compared to day 0 and ${ }^{b} p<0.001$ - as compared to day 30 (intragroup comparison), "(intergroup comparison)

muscle tone (i.e. Ashworth scale) also improved significantly $(p=0.011)$ in Group B as compared to Group A. However, other parameters did not $(p>0.05)$ show significant improvement and were found to be statistically the same between groups.

\section{Discussion}

The study hypothesized that Erigo tilt-table therapy would be more effective than conventional physiotherapy for early mobilization and rehabilitation in acute stroke patients. This hypothesis was found to be partially true as outcomes measures, QOL, NIHSS, muscle strength lower limb improved more in Group B. The improvement could be related to the fact that robotic tilttable rehabilitation may offer standardized, intensive and repetitive exercises, proper body weight support, with an appropriate sensory feedback amount and a controlled progressive verticalization. It was also found in another study that ERIGO training could be a valuable tool for the adaptation to the vertical position with a better global function improvement, as also suggested by the sensory-motor and vestibular system plasticity induction in post-stroke patients [14].

One study suggested that robotic verticalization maximizes the potential for longitudinal weight-bearing through the lower extremities in a position of hipextension/knee-extension/ankle-dorsiflexion, which is difficultly obtained in the physiotherapy verticalization setting. Moreover, robotic verticalization allows strengthening exercises using body weight shifting from one leg to the other, which is not easily carried out by severe post-stroke patients [14]. Some studies found that greater cerebral blood flow modulation during robotic verticalization in comparison to physiotherapy verticalization could further support plastic changes within sensory-motor areas and vestibular system, with the consequent motor and cognitive function amelioration [32, 33].

Rehabilitation on a tilt-table has been reported to be a useful way to mobilize severely impaired or noncooperating patients, since it improves circulation, prevents contractures, and increases pulmonary ventilation $[34,35]$. Our study supports the safety and effectiveness of robotic Erigo tilt table verticalization in bedridden post-stroke patients even in the acute phase.

Our study suggests that a reduction in motor impairments is greater after tilt-table exercises as it reduces long-term spasticity and improves strength. Our study supports another study that showed a significant increase in the EMG patterns of the extensors and flexors of the affected leg muscles during flexion and extension movements of both legs and clinical scores in patients undergoing the progressive task-oriented training on the tilt table compared to the other groups [36].

In our study, the Ashworth scores were less in the tilttable group when compared with conventional physiotherapy group initially after 30 days of intervention which shows that tilt-table therapy reduced spasticity. Another study has also showed that extensor spasms are reduced after tilt table standing [37]. Robot-based rehabilitation has been shown to improve motor performance by boosting brain plasticity $[38,39]$.

With respect to the hemodynamic effects of robotic verticalization, it has been shown that robotic tilt-tables are effective in preventing blood pressure drops [40], which may prevent orthostatic responses to verticalization by improving venous return and, hence, potentiating the cardiac output and the cerebral blood flow. In addition to improving orthostatic tolerance, robotic tilttable may also be an effective exercise tool in initiating 

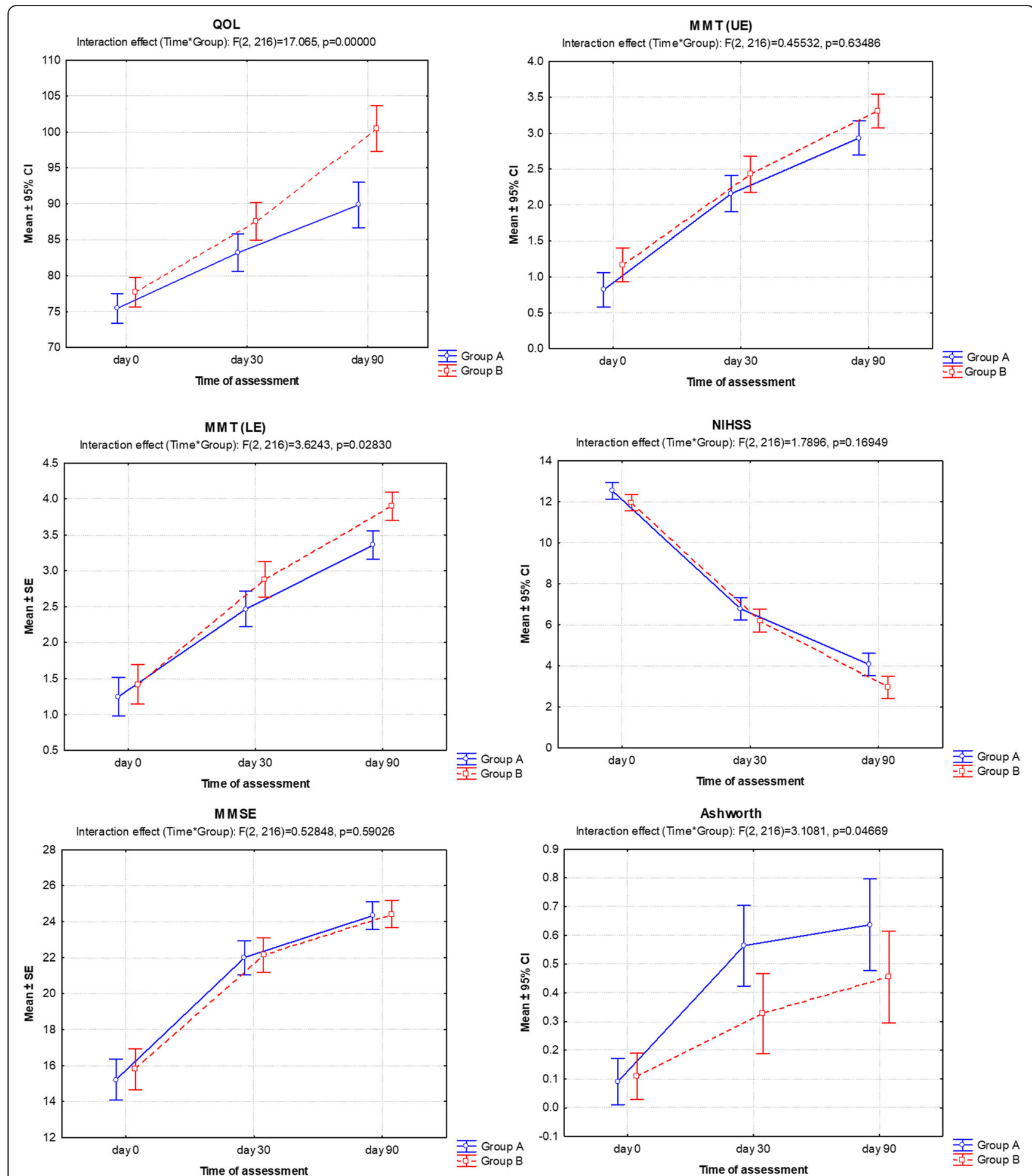

Fig. 2 Line graphs showing the mean outcome measure score of two groups over the periods with an interaction effect. Vertical bars denote 95\% confidence interval (CI). Group A: Conventional physiotherapy, Group B: Erigo tilt-table

the rehabilitation process at the earliest possible time point after stroke may minimize the reduction in aerobic fitness that occurs due to inactivity. It was also found that robotic tilt-table can be used to provide a strong training stimulus to complement conventional physiotherapy practices and serve the dual purpose of increasing orthostatic tolerance and attenuating the decline in aerobic fitness [41]. 
Table 4 Pre-treatment to post-treatment change in outcome measure score (Mean $\pm \mathrm{SD}$ ) between two groups at two different periods

\begin{tabular}{|c|c|c|c|}
\hline Outcome measure & $\begin{array}{l}\text { Group A } \\
(n=55)\end{array}$ & $\begin{array}{l}\text { Group B } \\
(n=55)\end{array}$ & $P$ value \\
\hline \multicolumn{4}{|l|}{$\mathrm{QOL}:$} \\
\hline Day 30 & $7.75 \pm 6.03$ & $9.87 \pm 6.84$ & 0.086 \\
\hline Day 90 & $14.38 \pm 8.83$ & $22.76 \pm 10.52$ & $<0.001$ \\
\hline \multicolumn{4}{|l|}{ MMT (UE): } \\
\hline Day 30 & $1.34 \pm 0.54$ & $1.26 \pm 0.46$ & 0.410 \\
\hline Day 90 & $2.11 \pm 0.76$ & $2.14 \pm 0.65$ & 0.851 \\
\hline \multicolumn{4}{|l|}{ MMT (LE): } \\
\hline Day 30 & $1.22 \pm 0.74$ & $1.46 \pm 0.69$ & 0.074 \\
\hline Day 90 & $2.11 \pm 0.90$ & $2.48 \pm 0.86$ & 0.030 \\
\hline \multicolumn{4}{|l|}{ NIHSS: } \\
\hline Day 30 & $-5.75 \pm 1.95$ & $-5.75 \pm 1.89$ & 1.000 \\
\hline Day 90 & $-8.45 \pm 1.89$ & $-8.98 \pm 1.62$ & 0.119 \\
\hline \multicolumn{4}{|l|}{ MMSE: } \\
\hline Day 30 & $6.78 \pm 3.26$ & $6.35 \pm 2.70$ & 0.446 \\
\hline Day 90 & $9.11 \pm 3.44$ & $8.62 \pm 2.93$ & 0.422 \\
\hline \multicolumn{4}{|l|}{ Ashworh: } \\
\hline Day 30 & $0.47 \pm 0.57$ & $0.22 \pm 0.46$ & 0.011 \\
\hline Day 90 & $0.55 \pm 0.69$ & $0.35 \pm 0.58$ & 0.104 \\
\hline
\end{tabular}

Group A Conventional physiotherapy, Group B Erigo tilt-table, QOL Quality of life, $M M T$ (UE) Manual muscle testing (upper extremity) or overall upper limb strength, MMT (LE) Manual muscle testing (lower extremity) or overall lower limb strength, NIHSS National institute of health stroke scale, MMSE Minimental state examination, Ashworth Ashworth scale-6

Although MMSE and muscle strength of the upper limb in both the groups improved equally, the other parameters improved more after tilt-table exercises. It has been suggested that verticalization may play a role in stimulating cortical areas involved in the trunk and lower limb control, so that differentiation and learned non-use can be contrasted [42]. Verticalization may actively contribute to enhancing cognitive performance through an increase in cerebral blood flow with consequent induction of cortical plasticity, especially in frontal lobes [43]. Robotic verticalization includes increased ventilation, increased arousal, improved weight-bearing of the lower limbs, and facilitation of antigravity exercise of the limbs [44].

Thus, Erigo tilt- table exercises can be practiced for the rehabilitation of acute stroke patients where early mobilization is difficult and delayed due to trunk instability, orthostatic hypotension, reduce vigilance and cooperation.

Although the study found beneficial effects many respects, it has several limitations such as lack of long duration follow-up and a larger sample size which can be answered in future studies.

\section{Conclusion}

The study concluded that both the Erigo tilt-table and conventional physiotherapy can to be beneficial and effective in the rehabilitation of acute stroke patients. This study also found that the tilt-table exercises were more beneficial in improving QOL, NIHSS, and muscle strength of the lower limb. The practitioner should consider Erigo tilt-table exercise to reduce the risk of complications and reduce impairments in stroke patients.

\section{Acknowledgements}

The authors acknowledge Indian Council of Medical Research, New Delhi for approval and funding of this extramural research project and Vice Chancellor of ' $X$ ' to conduct the research for the betterment of the patients. The authors wish to acknowledge patients for their supportive nature and cooperation.

\section{Authors' contributions}

SK and RY has been involved in drafting the manuscript or revising it critically for important intellectual content and given final approval of the version to be published. A has made substantial contributions to conception and design, or acquisition of data, or analysis and interpretation of data. All authors read and approved the final manuscript.

\section{Funding \\ Indian Council of Medical Research, New Delhi, India}

\section{Availability of data and materials}

The datasets used and/or analysed during the current study are available from the corresponding author on reasonable request.

\section{Ethics approval and consent to participate}

The present study has the approval of the Institutional Local Ethica Committee, Uttar Pradesh University of Medical Sciences and informed consent was obtained from all the participants.

\section{Consent for publication}

Not Applicable.

\section{Competing interests}

The authors declare that they have no competing interests.

\section{Author details}

'Department of Physiotherapy, UPUMS, Saifai, Etawah, UP 206130, India.

${ }^{2}$ Department of Neurology, UPUMS, Saifai, Etawah, UP, India.

Received: 22 November 2018 Accepted: 8 January 2020

Published online: 04 February 2020

\section{References}

1. Sacco RL, Kasner SE, Broderick JP, et al. An updated definition of stroke for the 21st century: a statement for healthcare professionals from the American Heart Association/American Stroke Association. Stroke. 2013;44(7): 2064-89.

2. Dittmer D, Teasell R. Complications of immobilization and bed rest. Part 1: musculoskeletal and cardiovascular complications. Can Fam Physician. 1993; 39:1428-32.

3. Bourdin G, Barbier J, Burlem JF, et al. The feasibility of early physical activity in intensive care unit patients: a prospective observational one-center study. Respir Care. 2010;55(4):400-7.

4. Burtin C, Clerckx B, Robbeets C, et al. Early exercise in critically ill patients enhances short-term functional recovery. Crit Care Med. 2009; 37(9):2499-505.

5. Frazzitta G, Valsecchi R, Zivi I, et al. Safety and feasibility of a very early verticalization in patients with severe traumatic brain injury. J Head Trauma Rehabil. 2015;30(4):290-2.

6. Rocca A, Pignat JM, Berney $L$, et al. Sympathetic activity and early mobilization in patients in intensive and intermediate care with severe brain 
injuries: a preliminary prospective randomized study. BMC Neurol. 2016; 16(1):169.

7. Luther MS, Krewer C, Müller F, et al. Comparison of orthostatic reactions of patients still unconscious within the first three months of brain injury on a tilt table with and without integrated stepping. A prospective, randomized crossover pilot trial. Clin Rehabil. 2008;22(12):1034-41.

8. Taveggia G, Ragusa I, Trani V, et al. Robotic tilt table reduces the occurrence of orthostatic hypotension over time in vegetative states. Int J Rehabil Res. 2015;38(2):162-6.

9. Frazzitta G, Zivi I, Valsecchi R, et al. Effectiveness of a very early stepping verticalization protocol in severe acquired brain injured patients: a randomized pilot study in ICU. PLoS One. 2016;11(7):e0158030.

10. Chi L, Masani K, Miyatani M, et al. Cardiovascular response to functional electrical stimulation and dynamic tilt table therapy to improve orthostatic tolerance. J Electromyogr Kinesiol. 2008;18(6):900-7.

11. Yoshida T, Masani K, Sayenko DG, et al. Cardiovascular response of individuals with spinal cord injury to dynamic functional electrical stimulation under orthostatic stress. IEEE Trans Neural Syst Rehabil Eng. 2013;21(1):37-46.

12. Pekna M, Pekny M, Nilsson M. Modulation of neural plasticity as a basis for stroke rehabilitation. Stroke. 2012;43(10):2819-28

13. Masiero $S$, Poli $P$, Rosati $G$, et al. The value of robotic systems in stroke rehabilitation. Expert Rev Med Devices. 2014;11(2):187-98.

14. Calabrò RS, Naro A, Russo M, et al. Do post-stroke patients benefit from robotic verticalization? A pilot-study focusing on a novel neurophysiological approach. Restor Neurol Neurosci. 2015;33(5):671-81.

15. Kumar S, Sharma VP, Negi MPS. Efficacy of dynamic muscular stabilization technique (DMST) over conventional technique in rehabilitation of chronic low back pain. J Strength Cond Res. 2009;23(9):2651-9.

16. Yadav R, Kumar S, Aafreen, et al. Robotic Erigo tilt table exercises versus conventional exercises in rehabilitation of hemiplegic patients - a pilot study. IJTR. 2018;25(9):475-80.

17. Bavikatte G, Gaber T. Approach to spasticity in general practice. Br J Med Pract. 2009;2(3):29-34

18. Ghai A, Garg N, Hooda S, et al. Spasticity - pathogenesis, prevention and treatment strategies. Saudi J Anaesth. 2013;7(4):453-60

19. Bordoloi K, Deka RS. Scientific reconciliation of the concepts and principles of rood approach. Int J Health Sci Res. 2018;8(9):225-34.

20. Eng JJ, Levins SM, Townson AF, et al. Use of prolonged standing for individuals with spinal cord injuries. Phys Ther. 2001:81(8):1392-9.

21. Ben $M$, Harvey $L$, Denis $S$, et al. Does 12 weeks of regular standing prevent loss of ankle mobility and bone mineral density in people with recent spinal cord injuries? Aust J Physiother. 2005;51(4):251-60.

22. Turner-Bowker DM, Bartley PJ, Ware JE Jr. SF-36 ${ }^{\oplus}$ health survey \& "SF" bibliography: third edition (1988-2000). Lincoln: Quality Metric Incorporated; 2002.

23. Kumar S, Sharma VP, Aggarwal A. Correlation and regression among pain, physical strength, functional ability, quality of life and sexual frequency in low back pain. J Musculoskelet Res. 2010;13(4):177-85.

24. Cohen JW, Ivanova TD, Brouwer B, et al. Do performance measures of strength, balance, and mobility predict quality of life and community reintegration after stroke? Arch Phys Med Rehabil. 2018;99(4):713-9.

25. Gunaydin R, Karatepe AG, Kaya T, et al. Determinants of quality of life (QOL) in elderly stroke patients: a short term follow up study. Arch Gerontol Geriatr. 2011;53(1):19-23.

26. Ciesla N, Dinglas $V$, Fan $E$, et al. Manual muscle testing: a method of measuring extremity muscle strength applied to critically ill patients. J Vis Exp. 2011;12(50):26-32.

27. Wist S, Clivaz J, Sattelmayer M. Muscle strengthening for hemiparesis after stroke: a meta-analysis. Ann Phys Rehabil Med. 2016;59(2):114-24.

28. Williams LS, Yilmaz EY, Lopez Yunez AM. Retrospective assessment of initial stroke severity with the NIH stroke scale. Stroke. 2000;31(4):858-62.

29. Zwecker $M$, Levenkrohn S, Fleisig $Y$, et al. A mini-mental state examination, cognitive FIM instrument, and the Loewenstein occupational therapy cognitive assessment: relation to functional outcome of stroke patients. Arch Phys Med Rehabil. 2002;83(3):342-5.

30. Gregson JM, Leathley MJ, Moore AP, et al. Reliability of the tone assessment scale and the modified ashworth scale as clinical tools for assessing poststroke spasticity. Arch Phys Med Rehabil. 1999;80(9):1013-6.
31. Ansari NN, Naghdi S, Arab TK, et al. The interrater and intrarater reliability of the modified Ashworth scale in the assessment of muscle spasticity: limb and muscle group effect. NeuroRehabilitation. 2008;23(3):231-7.

32. Raethjen J, Govindan R, Binder S, et al. Cortical representation of rhythmic foot movements. Brain Res. 2008;1236:79-84.

33. Wieser M, Haefeli J, Butler $L$, et al. Temporal and spatial patterns of cortical activation during assisted lower limb movement. Exp Brain Res. 2010;203(1): 181-91.

34. Chang AT, Boots R, Hodges PW, et al. Standing with assistance of a tilt table in intensive care: a survey of Australian physiotherapy practice. Aust J Physiother. 2004;50(1):51-4.

35. Cumming TB, Thrift AG, Collier JM, et al. Very early mobilization after stroke fast-tracks return to walking: further results from the phase II AVERT randomized controlled trial. Stroke. 2011;42(1):153-8.

36. Kim CY, Lee JS, Kim HD, et al. Lower extremity muscle activation and function in progressive task-oriented training on the supplementary tilt table during stepping-like movements in patients with acute stroke hemiparesis. J Electromyogr Kinesiol. 2015;25(3):522-30.

37. Bohannon RW. Tilt table standing for reducing spasticity after spinal cord injury. Arch Phys Med Rehabil. 1993;74(10):1121-2.

38. Duret C, Courtial O, Grosmaire AG, et al. Use of a robotic device for the rehabilitation of severe upper limb paresis in subacute stroke: exploration of patient/robot interactions and the motor recovery process. Biomed Res Int. 2014;2015(482389):1-7.

39. Pellegrino $G$, Tomasevic $L$, Tombini $M$, et al. Inter-hemispheric coupling changes associate with motor improvements after robotic stroke rehabilitation. Restor Neurol Neurosci. 2012;30(6):497-510.

40. Kuznetsov AN, Rybalko NV, Daminov VD, et al. Early post-stroke rehabilitation using a robotic tilt-table stepper and functional electrical stimulation. Stroke Res Treat. 2013;2013:94605-6.

41. Craven CTD, Gollee H, Coupaud S, et al. Investigation of robotic-assisted tilttable therapy for early-stage spinal cord injury rehabilitation. J Rehabil Res Dev. 2013:50(3):367-78.

42. Pittaccio S, Zappasodi F, Tamburro G, et al. Passive ankle dorsiflexion by an automated device and the reactivity of the motor cortical network. Conf Proc IEEE Eng Med Biol Soc. 2013;2013:6353-6.

43. Reinstrup $P$, Ryding $E$, Algotsson $L$, et al. Effects of nitrous oxide on human regional cerebral blood flow and isolated pial arteries. Anesthesiology. 1994; 81(2):396-402

44. Dean E, Ross J. Oxygen transport: the basis for contemporary cardiopulmonary physical therapy and its optimization with body positioning and mobilization. Phys Ther Prac. 1992;1:34-44.

\section{Publisher's Note}

Springer Nature remains neutral with regard to jurisdictional claims in published maps and institutional affiliations.
Ready to submit your research? Choose BMC and benefit from:

- fast, convenient online submission

- thorough peer review by experienced researchers in your field

- rapid publication on acceptance

- support for research data, including large and complex data types

- gold Open Access which fosters wider collaboration and increased citations

- maximum visibility for your research: over $100 \mathrm{M}$ website views per year

At $\mathrm{BMC}$, research is always in progress.

Learn more biomedcentral.com/submission 